\title{
Morphological Feature Detection *
}

\author{
J. Alison Noble \\ Robotics Research Group \\ Department of Engineering Science \\ University of Oxford
}

We describe investigations applying grey-scale mathematical morphology to the problem of feature detection. We show how a combination of morphological operators can be interpreted in terms of the differential geometrical characteristics of the intensity surface. This is significant in that it provides insight into how morphological operators manipulate image data in a manner that has no parallel in traditional convolutionbased image processing. Results using a simple morphological boundary detector compare favourably with the output of a normal edge detector such as the Canny operator. However, boundary detection differs in two important respects; the performance is generally better in regions of high image curvature and image junction information remains explicit. We provide experimental evidence to support these claims. An image description is only of use if it is an aid to image understanding. We conclude with a brief discussion of a morphologically derived scheme based on boundary surface features and indicate how such a description provides potentially powerful constraints for correspondence algorithms.

\section{Introduction}

This paper is concerned with the application of grey-level morphology to low-level feature detection in intensity images for computer vision purposes. To the author's knowledge, there has only been one other published work specifically on this subject. Lee, Haralick and Shapiro discuss the implementation of a morphological edge detector [10]. They derive a 'blur-minimum' edge detector based on simple morphological operations and compare its performance to that of the cubic facet second directional derivative edge detector (2DD) [5] and a number of threshold/enhancement operators. Although the morphological edge detector's performance is comparable to that of the $2 \mathrm{DD}$, the development of the operator appears rather ad hoc and does not seem to offer a natural extension to design detectors for other image features.

In the past two decades or so, the theoretical basis for much research into edge detection has focussed on exploiting the algebraic structure of images. The Marr-Hildreth Laplacian of a Gaussian and the Canny operator are probably the most well-known edge operators of this type. However, the performance of a Canny operator in regions of high image curvature

*This is a summary of a paper in preparation (texture, corners etc) and at image junctions is both inconsistent and unpredictable. Why this is so is quite simple. Edge detectors are designed for 1D 'events'. Corners and junctions clearly do not have such a simple image structure (see for example, the ' $\mathrm{T}$ '-junction in figure 1).

The structure of corners and junctions contain rich geometric information about the imaged scene, giving information about such things as object surface intersections, reflectance discontinuities and illumination discontinuities. However, with the exception of the ' $\mathrm{L}$ '-junction [4], it is not possible to formalise a general model for such image structures in terms of differential parameters. In a previous paper [18], I showed how corners and junctions could be characterised in terms of the differential geometry of a facet model. Clusters of elliptic and hyperbolic points were found to be good indicators of such two-dimensional 'events' in real images. It is the idea of grouping neighbourhood responses which is important here. Responses to second differential operators reflect their source of origin. Random noise generates a spurious response, whereas neighbourhood support provides evidence of genuine two-dimensional image structure. Finding specific image structure suggested looking at mathematical morphology as an alternative means to both detect and describe junctions. This paper reports on some of these investigations.

The foundations of the mathematics of morphology are attributed to Matheron [14] and Serra [22] in the mid 70's. Later work, most notably by Sternberg [24], has extended the analysis from binary to grey-level morphology. Despite applications in industry, until recently the academic vision community has shown little interest in exploiting morphological techniques. Most work on morphological processing has focused on binary images. Surprisingly, grey-level morphology has received less attention, even though the morphological transformations frequently admit of useful geometric interpretation. Relating these inherently nonlinear 'image re-shaping' operations to more familiar techniques of image analysis remains an important gap in our present understanding of this image processing technique. Towards this goal, Maragos considers some relationships between nonlinear and linear filters in $[12,13]$. This paper is concerned with differential geometrical interpretations of morphological operations.

Section 1 considers boundary detection and discusses aspects of morphological operations which make them well suited for edge and boundary detection. We describe an apparently simple boundary detector to show how our intu- 
itions, in this case about the meaning of an edge, can be translated into the language of mathematical morphology. Key concepts relating differential and morphological structures turn out to be parallel and canal surfaces. We review some of the relevant properties of these mathematical surfaces and show how they can help us to characterise boundary points. The detection of valleys, ridges and corners is considered in Section 2, where it is shown how and why the operations of closing and opening can be interpreted as measures of image curvature. This leads us to comment briefly on the connection between mathematical morphology and the blending of surfaces in solid modellers in Section 3. An image description is only of use if it is an aid to image understanding. In Section 4 we present experimental results to show how a scheme based on boundary surface features has potential for providing powerful topological constraints for correspondence algorithms. Edge maps based on this scheme are shown to compare favourably with those derived from the Canny operator. However more significantly, junction information remains explicit. We conclude the account by indicating some of the future directions for our investigations.

\section{Boundary Detection ${ }^{1}$}

In this section we assume ideal, noise free imagery, and as usual only consider the case of surfaces with one dimensional (1D) discontinuities.

Broadly speaking, physical edges give rise to $1 \mathrm{D}$ discontinuities in the intensity image surface [8]. As is well known, this suggests that edges can be found by locating local maxima in the first directional derivative or equivalently, zeros in the second directional derivative taken in the direction of the gradient. A number of edge operators are based on this principle $[5,2,23]$.

We identify several practical problems with this approach. 'Blurring' introduced by imaging systems is usually ignored in theoretical analysis and considered a hindrance in practice, yet it gives a characteristic shape to the grey level function in the vicinity of boundaries which often has a direct correlation to the source of origin. 'Edge' detectors do not perform reliably at image junctions where the local surface clearly does not have a $1 D$ structure. Further, strictly speaking as we are dealing with digital data, we require regularisation ${ }^{2}$ to make the surface differentiable. However, the detail of many powerful image constraints, such as ' $\mathrm{T}$ ', and ' $\mathrm{Y}$ '- junctions will be destroyed by such processing.

Morphological techniques, provide a way of filtering images that potentially avoids these criticisms. Because the language of mathematical morphology is based on transforming images treated as sets, the mathematical problem of defining boundaries is well-posed. Further, there is a clear distinction between the role of derivatives and gradients in the 'traditional' treatment of surfaces and that used in the context of mathematical morphology.

\subsection{Morphological Boundary Points}

A boundary $\partial X$, in the topological sense can be defined as the set of points for which all neighbourhoods intersect both

\footnotetext{
${ }^{1}$ Space limitations mean that some familiarity with mathematical morphology has been assumed. [6] is a good tutorial paper on the subject

${ }^{2}$ often Gaussian smoothing but not necessarily
}
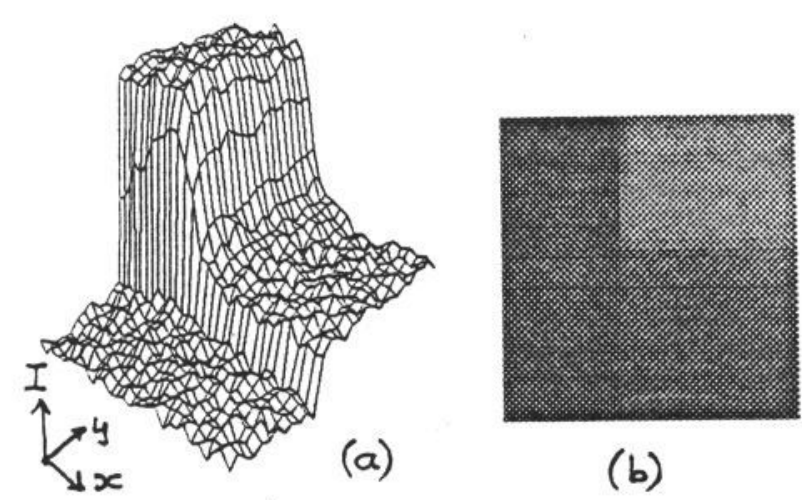

(b)

Figure 1: Differential operators manipulate the 2D projection (b) of the intensity image surface (a). This explains why they can't reliably find junctions.

the set $X$ and its complement $X^{c}$. Translated into mathematical morphology (details can be found in [21]) this leads to the following definition:

Morphological Boundary: $A$ morphological boundary $\partial X_{m}$ of $a$ set $X$ is defined as the set difference of the dilation and erosion of a set $X$ by a spherical structuring element $B_{r}$.

$$
\partial X_{m}=\left(X \oplus B_{r}\right)-\left(X \ominus B_{r}\right)
$$

A similar definition is commonly used in the morphological community as the basis for gradient estimate edge operators, except that disc or line elements are used in place of the sphere. However this apparent similarity is misleading. A morphological boundary as defined above, is not what we normally regard as an edge. That is, morphological boundary points include regions (smooth surface patches), edges (singularity curves) and junctions (singularity points). The explanation for this difference is as follows. Grey-level morphological operators are $3 \mathrm{D}$ operators in that they work with the image surface. Differential operators manipulate the 2D projection which explains why it is nontrivial to find junctions with them (figure 1). To a differential operator, the notion of a step and a corner, or a ridge ${ }^{3}$ and a sharp corner are distinct, and require quite different processing. However, to an isotropic grey-level morphological operator they are treated the same. For example, an ' $\mathrm{L}$ '-junction is like the top half of a step edge turned on its end, and a sharp corner is interpreted in the same way as a ridge (figure 2). A morphological operation suitably chosen to respond to the characteristics of a ridge will also respond to a corner. Clearly, to use morphological operators successfully, we need to understand how the surface is 'seen' by a morphological operator, and based on this, interpret the response accordingly. We now show that this behaviour has a direct interpretation in terms of the differential geometry of the underlying surface.

\footnotetext{
${ }^{3}$ Here, a ridge is taken to be a location in a digital (not necessarily intensity) image, where a simply connected sequence of pixels have values significantly larger than their neighbours.
} 


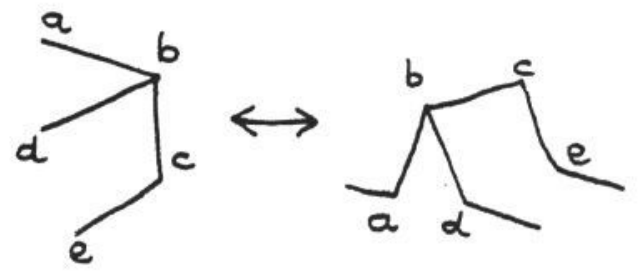

Figure 2: A grey-level spherical structuring element treats sharp corners like ridges

\subsection{Parallel Surfaces}

A surface $\mathrm{S}_{\| r}$ defined by moving a distance $r$ (the offset), along the normal $\mathrm{N}$ to a smooth surface $\mathrm{S}_{\circ}$ at each point of the surface, is parallel to the original and has the form:

$$
\mathrm{S}_{\| r}=\mathrm{S}_{\circ}+r \mathrm{~N}
$$

For every continuous surface there are an infinite number of parallel surfaces each of which has the same surface normal as the original (figure 3). Weatherburn [28] has shown that a family of such parallel surfaces exists provided the curl of the surface normal is zero. Our interest in this special mathematical surface derives from the fact that the dilation and erosion of a smooth surface by a spherical structuring elements of radius $\rho$ defines two parallel surfaces at distance $\pm \rho$ from the original. The set difference between the two gives a set of surface boundary points which we call a smooth patch (of strength $2 \rho$ ) and satisfies the following definition:

Smooth $\mathrm{Patch}: A$ smooth patch is a connected set of points in $\partial X$ that have two dimensional neighbourhoods in $\partial X$ such that $\partial X$ is $C^{1}$ continuous (i.e. have continuous surface normal).

Even for a smooth surface, singularities in the parallel surfaces may exist. This will always arise for some value of $\rho$ if the curl of the surface normal is non-zero. For, let $\kappa_{1,2}$ be the principal curvatures of the surface at a point $\left(x_{0}, y_{0}\right)$. Then the principal curvatures on the parallel surfaces at distance $\pm r$ can be shown to be:

$$
\kappa_{1 \pm r}=\frac{\kappa_{1}}{1 \pm r \kappa_{1}}, \kappa_{2 \pm r}=\frac{\kappa_{2}}{1 \pm r \kappa_{2}}
$$

We see that when one of the principal curvatures becomes equal in magnitude to $1 / r$, the curvature becomes infinite and there is a singular point. A sphere of radius $\rho=r$, will 'lose contact' at such points, and the set difference dilation-erosion will be greater than $2 \rho$. Singularities in this case result from characteristics of the operator and not the underlying surface. Singularities arising from features of the surface (ridges and step changes) give rise to singular curves $C$ defined as follows:

Singular Curve $C: A$ singular curve $C$ of $\partial S$ is a connected set of points $x$ of $\partial S$ that have one dimensional neighbourhoods such that $C$ is continuous (i.e. have continuous unit tangent).

In a $3 \mathrm{D}$ polyhedral scene, singular curves correspond to edges of objects without their end points. In intensity images, all 1D features (i.e. steps, ridges, valleys, thin bars) are examples. To distinguish between these cases, (after all image interpretation is the objective behind feature point detection, [19]), we need to derive further constraints based on the neighbourhood surface characteristics about the boundary curve. One way to do this using morphological operations

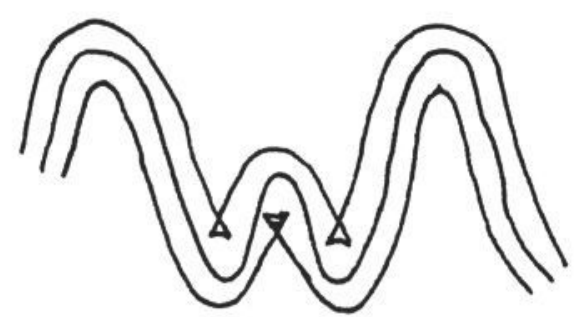

Figure 3: Parallel Surfaces: $1 D$ profile showing two offset curves and six cusps

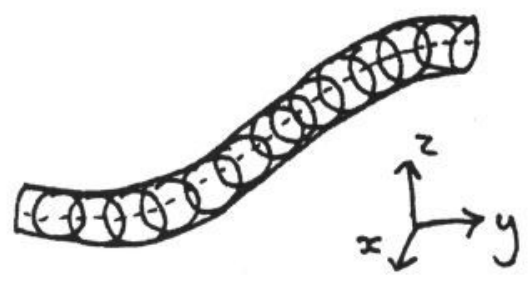

Figure 4: A canal surface of spheres of constant radius

is by considering the surface generated by the dilation-erosion difference transformation.

\subsection{Canal Surfaces}

A canal surface is defined as the envelope of a family of spheres, which may have continuously varying radii, with centres lying on a smooth curve. The special case of the constant radius canal surface can be obtained by sweeping a circular cross-section along the curve (figure 4). Each sphere is tangent to the envelope along a circle, such a curve being a line of curvature of the surface. This type of surface is also known as a right circular constant generalised cylinder or a tube. We can easily show that the dilation and erosion of a step edge by a spherical structuring element gives two points that belong to a circle in the normal plane of the curve $C$. That is, step edges are characterised by the generation of points that lie on a canal surface of a boundary singularity curve under the dilation-erosion difference operation. The same can not be said to be true for a singularity curve arising from a ridge (valley or sharp corner). We consider a way to describe the surface geometry of these features using morphological transformations in the next section.

The complete morphological boundary point categorisation requires the definition of a singular point.

Singular Point:Points of $\partial S$ that do not belong to a smooth surface patch or singular curve are singular (Morse) points of the boundary.

In a polyhedral world these correspond to object vertices. In intensity images, ' $T$ '-, ' $\mathrm{Y}$ '- and ' $\mathrm{W}$ '- junctions are examples.

To summarise, boundary points of a surface can be defined in terms of the set difference dilation-erosion to be of one of three distinct types. That is a boundary point must lie either in a smooth surface patch, or in a singular curve or be a singular point. Defining a boundary detector in this way proves a good way to find junctions and step discontinuities in intensity images. For the detection of ridges, valleys and sharp corners a different operator is required. 


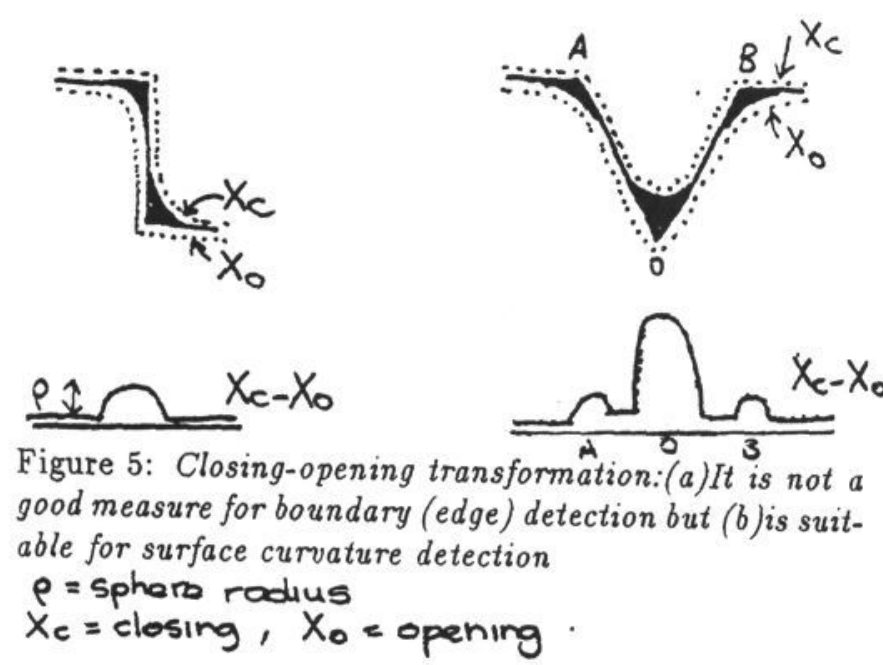

\section{Valleys, Ridges, Corners}

A natural extension to the analysis given in the previous section is to consider the effect of the opening and closing operations on intensity features. We can visualise the spherical opening operation as rolling a sphere under the surface and only keeping points where the sphere touches the surface [25]. The dual operation of closing rolls the sphere over the topside of the surface. Figure 5a shows the spherical closing, opening and their difference profile for a step intensity change. Evidently the closing-opening difference is not a good measure for boundary (or edge) detection because the requirement of accurate boundary localisation (small $\rho$ ) conflicts with that for good detection ( $\rho$ large). Applying the same transformations to a valley (or ridge) figure $5 \mathrm{~b}$, it is clear that the effect is to give a maximum response at high surface curvature points of the intensity profile. For an acute corner of angle $2 \theta(0<2 \theta<\pi)$ we see that for a given sphere radius $\rho$ the amount of the surface cut off is proportional to the acuteness of the angle (roughly speaking the curvature [1]), and the minimum distance from the apex of the corner to the opened surface is $\rho(\operatorname{cosec} \theta-1)$ (figure 6$)$. This suggests that the closing-opening difference can be used for valley (or ridge) detection and leads us to the following definition:

Ridge and Corner Detector: $A$ morphological ridge (valley) and sharp corner detector is suitably defined by the set difference of the closing and opening of the image surface by a spherical structuring element.

A ridge and sharp corner are similar entities as far as a morphological operator is concerned, for in both cases the operator responds to the singularity curve in the surface. The essential difference is, that at an ' $L$ '-junction the maxima appear as an isolated peak. In the case of a ridge, we get a series of such high responses giving a ridge-line. We can use this difference in local topography to distinguish between the two surface 'events'.

\section{Offsets and Blends}

Offsetting operations, also known as expanding and shrinking, have been used for a number of practical purposes including collision-free path planning, mould design and numerical control (NC) machine tool design. For the case of a ball-ended cutter, the tool centre traces a path at a constant distance (called the offset) from the original. This defines a new parallel surface (see section 2.1 ) parallel to this surface. It has the same surface normal as the original, and for this reason this process is also known as n-offsetting. We can define a set of planar parallel curves for a $2 D$ surface profile by n-offsetting

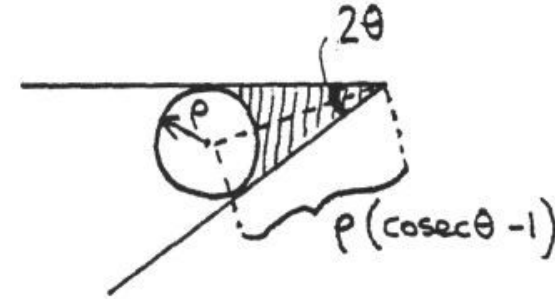

Figure 6: Measuring curvature of a sharp corner using continuous morphological operators

with a cylindrical (milling) cutter. Offsets of this type have been studied in some detail particularly with regard to problems in regions of high curvature $(2 \mathrm{D}$ or $3 \mathrm{D})$ or at sharp corners, where the profile followed may have a cusp and be self-intersecting (figure 3). A number of heuristic algorithms have been proposed to account for these special cases [27], but there is no mathematical definition for piecewise smooth parallel curves and surfaces.

Algorithms to enable blending facilities to be included in dual-representation solid modellers containing both Computational Solid Geometry (CSG) and Boundary (Brep) representations have been derived in terms of spherical morphological transformations [21]. Offsetting operations, (shrinking/expanding) are special cases of Minkowski addition and subtraction and correspond to the morphological operations of dilation and erosion with a sphere; spherical opening and closing define the rounding and filleting (constant-radius blending) transformations. Of course, in solid modellers the underlying assumptions as to surface forms, imaging conditions and overall goals are quite different from those in computer vision. However, the major geometric problems are common to both. How can we define representations for boundaries or classify surface boundary points as belonging to regions (faces), edges or junctions (vertices)? How do we cope with interference of blends, and account for loss in detail and incorrect termination of edges entering junctions? All these problems stem from the inability to derive adequate algebraic or geometric descriptions for the features we are trying to model.

\section{Results and Extensions}

The algorithms described have been implemented in LISP on a HP 9000 (Bobcat) using real images. A low 'noise' threshold of six pixels was used throughout (i.e. 2 pixel diameter sphere plus an allowance for imaging-device and other sources of noise of \pm 2 pixels ). The digital sphere approximation has radius 1 pixel (i.e. $3 \times 3$ neighbourhood) and can be represented in umbrae notation as:

$$
\begin{aligned}
& \text { U }\left[\begin{array}{lll}
0 & 1 & 0
\end{array}\right] \\
& {\left[\begin{array}{lll}
1 & 1 & 1
\end{array}\right]} \\
& {\left[\begin{array}{lll}
0 & 1 & 0
\end{array}\right]}
\end{aligned}
$$

(In this representation the values $1,0,-1$ represent 'on' pixel positions in top, middle and bottom layers of a $3 \times 3 \times 3$ cube.)

\subsection{Feature Detection}

1. Figure 7 shows some of the different junction types extracted using the dilation-erosion difference transformation on the first frame of the Plessey widget sequence [7]. The new surface defined by this transformation we call the boundary surface $[x, y, k]$, where $k$ is the boundary strength and $x, y$ 
have their usual meaning. Boundary points appear as clearly defined ridges and junction information remains explicit.

2. Figure $8 \mathrm{a}$ shows the boundary surface for an acute corner taken form the same widget image. The closing-opening difference transformation response is shown in figure $8 \mathrm{~b}$. The peak response agrees with the theoretical prediction given in section 2.

\subsection{Image Descriptions}

3. Although this paper is not concerned specifically with extracting ridges and contours from the boundary surface, to illustrate the relative robustness of the general boundary detection technique we compare qualitatively the output of a simple morphological ridge detector with the Canny operator. Figure 9 shows a complex scene (both in terms of intensity structure and noise). Figure $9 \mathrm{a}$ shows the result of applying the spherical opening-residue transformation [15] to the boundary surface. A Canny output on the same image is shown in figure $9 \mathrm{~b}$. Canny derived his optimal straight edge operator using variational calculus techniques. The principles behind the morphological boundary detector are quite different and based on preserving the connectivity in grey-scale images. Bearing in mind that there is no explicit smoothing or an edge-linking process and the current implementation is single-scaled, the morphological result compares favourably with the Canny response. Where the morphological boundary detector comes into its own is at image junctions and in highly curved regions where clearly the step edge assumption does not hold. Evidently, there is considerable room for improvement on the current implementation. We intend to report on studies of noise analysis and more sophisticated ridge detection/structural filtering methods [20] in a sequel to this paper.

4. Figure 10 illustrates the result of applying the boundary/ridge algorithms to the first frame of a hand motion sequence. The boundary surface response (figure 10b) is displayed as intensity; namely, a large response is displayed with a darker value. Edges and junctions in the original grey-level image appear as distinct ridges and peaks in the boundary surface. That is, morphological transformation has enhanced topological structures in the image. This example has been chosen to illustrate two distinguishing features of the boundary surface representation. First, the cusp between the thumb and first finger gives an abruptly terminating boundary end. This would not be detected by a normal edge or region detector, since edge detection algorithms generally 'follow' closed connected edge contours while region-based detectors do not allow for internal boundaries.

There is a close relationship between gradient-based edges, and the projection of the ridge lines on to the $(x, y)$ plane. These give information about the spatial relationships between objects. The ridge lines can be thought of as $3 \mathrm{D}$ space contours in the boundary surface domain and provide a different type of image description. An interesting question is, can the variation of boundary strength along these contours (as along the fingers) be used to infer additional information about the bounded surfaces? Nackman [16] has shown this is true in theory for smooth surfaces. This behaviour has been shown to be useful for describing images [9], and as the radius function of a three dimensional symmetric axis [17]. The key point is that singularity curves provide information about more than one type of change in the original image. The type of boundary information we chose to use (ridge projection, ridge-lines or both) will be determined by vision task. Whether and how we can use this information to augment normal edge-based image descriptions suggests a further direction for investigation.

The fact that topological descriptors can be derived from the boundary surface representation suggests morphological processing may be suited for providing topological matching constraints for use in higher vision reasoning. For example, it is well known that a major weakness of many current stereo and motion correspondence schemes is that ambiguities are made more severe by added and missing information due to noise, occlusion and errors in the input map - which is most noticeable in highly curved regions and at image junctions. Also, in line drawing interpretation, despite much activity directed towards (geometrically) cataloguing junctions of both polyhedral and curved objects (for example $[3,11,26]$ ), results on real edge data have been disappointing. The lack of robustness of these schemes to improperly classified and missing junctions remains a major source of frustration. Our experimentation seems to indicate that morphological techniques offer a possible methodology to provide reliable junction information. We hope to report on morphological junction classification using real data in future work.

\section{ACKNOWLEDGEMENTS}

I am grateful to Mike Brady for many discussions about the ideas described in this paper and helpful comments on presentation. Thanks also to Andrew Blake, Andrew Brint, Dave Forsyth, John Knapman and Andrew Zisserman for their help in a variety of ways. The support of the Science and Engineering Research Council and IBM UK is acknowledged.

\section{References}

[1] M. Brady, J. Ponce, A. Yuille, and H. Asada. Describing Surfaces. Comput. Graphics Image Processing, 32:1-28, 1985.

[2] J.F. Canny. Finding Edges and Lines. Technical Report Tech.Rep. 720, Massachusetts Inst. Technol., 1983.

[3] I. Chakravarty. A Generalized Line and Junction Labelling Scheme with Applications to Scene Analysis. IEEE Trans. Pattern Anal. Machine Intel., PAMI1(2):202-205, 1979.

[4] L. Dreschler and H.H. Nagel. Volumetric model and 3$\mathrm{D}$ trajectory of a moving car derived from monocular TV-frame sequence of a street scene. In Proc. IJCAI, pages 692-697, 1981.

[5] R.M. Haralick. Digital Step Edges from Zero-crossings of Second Directional Derivatives. IEEE Trans. Pattern Anal. Machine Intell., PAMI-6(1):58-68, 1984.

[6] R.M. Haralick, S.R. Sternberg, and X. Zhuang. Image Analysis using Mathematical Morphology. IEEE Trans. Pattern Anal. Machine Intell.,, PAMI-9(4):532$550,1987$.

[7] C.G. Harris and J.M. Pike. 3D Positional Integration from Image Sequences. In Proc. Alvey Vision Conference, Cambridge,England, 1987.

[8] B.K.P Horn. Understanding Image Intensities. Artificial Intelligence, 8:201-231, 1977. 
[9] J.J. Koenderink and A.J. van Doorn. The Structure of 2D Scalar Fields with Applications to Vision. Biol. Cybern., 33:151-158, 1979.

[10] Lee,J.S.J. and Haralick,R.M. and Shapiro,L.G. Morphological Edge Detection. Int. J. Robotics and Automation, RA-3(2):142-156, 1987.

[11] J. Malik. Interpreting Line Drawings of Curved Objects. Int. J. Computer Vision, 1:73-103, 1987.

[12] Maragos,P. and Schafer,R.W. Morphological FiltersPartI: Their Set-Theoretic Analysis and Relations to Linear Shift-Invariant Filters. IEEE Trans. Acoust., Speech, Signal Processing, ASSP-35(8):1153-1169, 1987.

[13] Maragos,P. and Schafer,R.W. Morphological FiltersPartII: Their Relations to Median,Order-Statistic, and Stack Filters. IEEE Trans. Acoust., Speech, Signal Processing, ASSP-35(8):1170-1184, 1987.

[14] G. Matheron. Random Sets and Integral Geometry. Wiley, New York, 1975.

[15] Meyer,F. Iterative Image Transformations for an Automatic Screening of Cervical Smears. J. Histochemistry and Cytochemistry, 27(1):128-135, 1979.

[16] L.R. Nackman. Two-dimensional Critical Point Configuration Graphs. IEEE Trans. Pattern Anal. Machine Intel., PAMI-6(4):442-449, 1984.

[17] L.R. Nackman and S.M. Pizer. Three-Dimensional Shape Description Using the Symmetric Axis Transform 1:Theory. IEEE Trans. Pattern Anal. Machine Intel., PAMI-7(2):187-202, 1985.

[18] J. Alison Noble. Finding Corners. Image and Vision Computing, 6(2):121-128, May 1988.

[19] J. Ponce and M. Brady. Towards a Surface Primal Sketch. Technical Report A.I. Memo 824, MIT A.I Lab., 1985.

[20] M. Richetin, P. Saint-Marc, and J.T. Lapreste. Describing Greylevel Textures through Curvature Primal Sketching. In Proc. of IEEE Int. Conf. ASSP, Tokyo, April 1986.

[21] J.R. Rossignac. Blending and Offsetting Solid Models. Technical Report, Univ. Rochester, N.Y., July 1985.

[22] J. Serra. Image Analysis and Mathematical Morphology. Academic Press, 1982.

[23] L. Spacek. The Computation of Visual Motion. PhD thesis, Univ. Essex, Colchester, 1984.

[24] S.R. Sternberg. Language and architecture for parallel image processing. Robotica, 2:33-40, 1984.

[25] Sternberg,S.R. Biomedical Image Processing. Computer, 16(1):22-34, January 1983.

[26] K. Sugihara. Mathematical structure of line drawings of polyhedra - towards man-machine communication by means of line drawings. IEEE Trans. Pattern Anal. Machine Intel., (4):458-469, 1982.

[27] W. Tiller and E.G. Hanson. Offsets of two-dimensional profiles. IEEE Computer Graphics and Applications, 4(9):36-46, September 1984.

[28] C.E. Weatherburn. Differential Geometry of Three Dimensions. Cambridge Univ. Press, 1947.
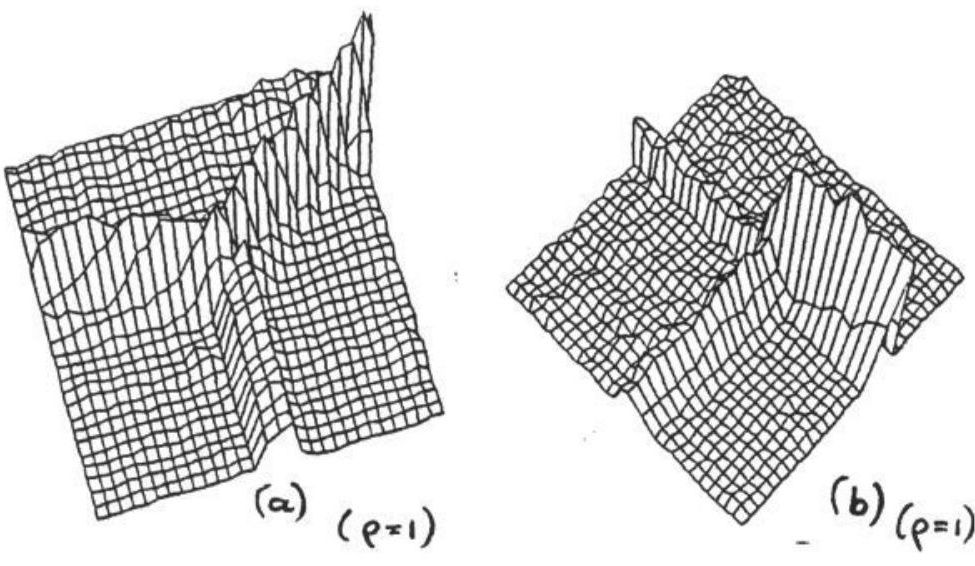

Figure 7: Morphological Boundary Detection of the vertices of a widget: (a) ' $Y$ ' junction, and (b) ' $T$ ' junction.
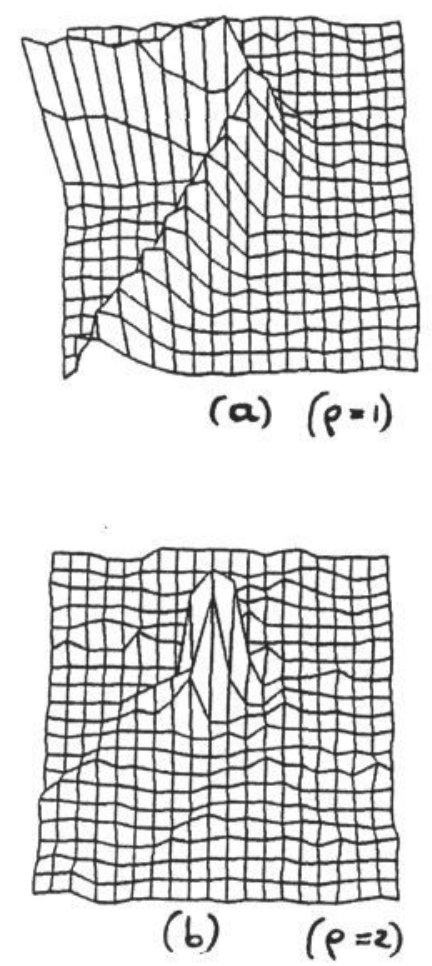

Figure 8: (a) Morphological boundary detection response at a sharp corner (b) Corner detection using the closing-opening difference transformation 
(a)

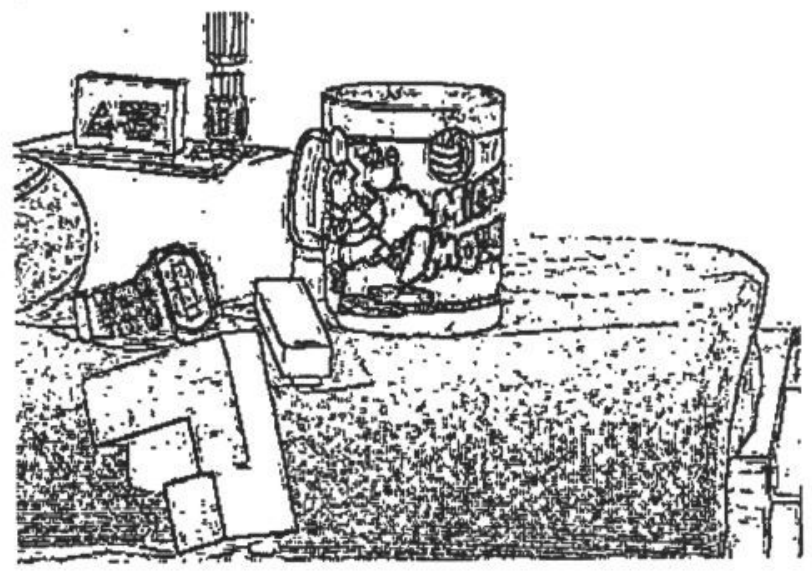

(b)

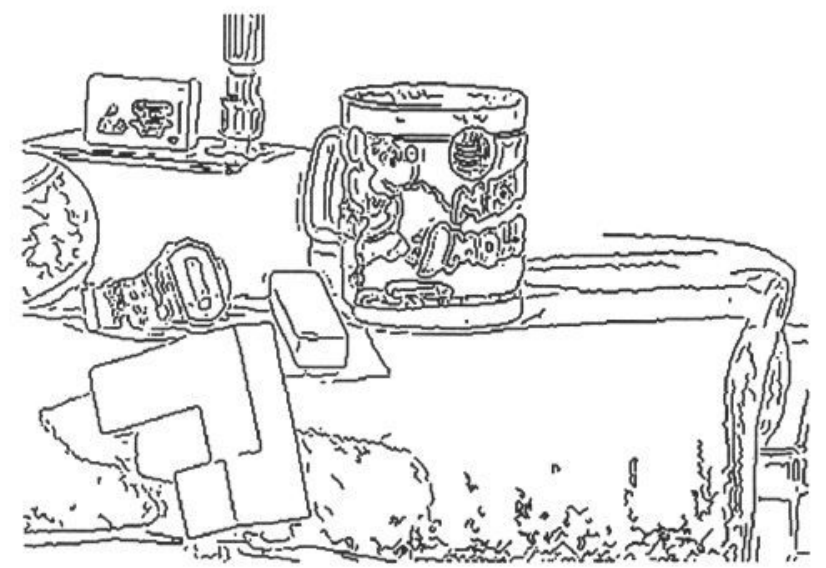

Figure 9: 'Widget Revisited':(a) Morphological Edge Response;(b) Canny output

(a)

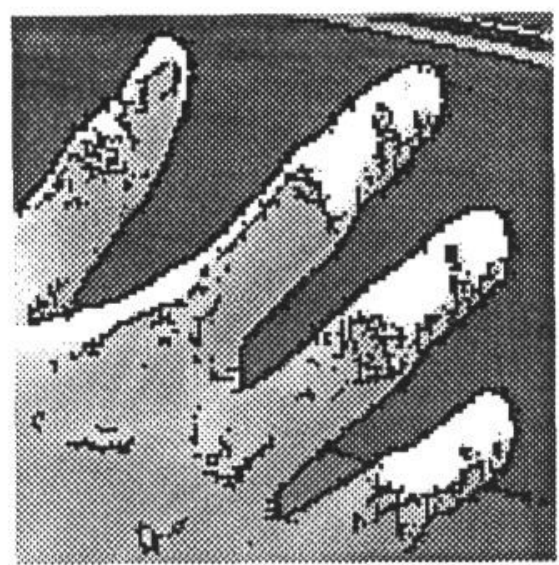

(b)

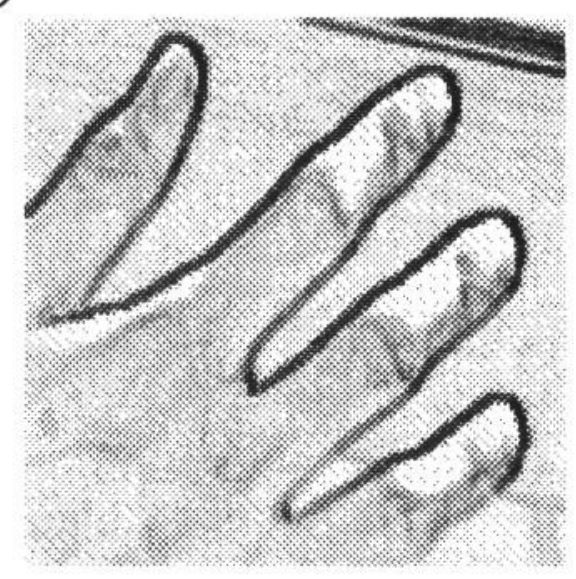

Figure 10: Hand Image: (a) Original image with boundary points superimposed; (b) Boundary surface 
